\title{
Rodlike localized structure in isotropic pattern-forming systems
}

\author{
Ignacio Bordeu* \\ Departamento de Física, Facultad de Ciencias, Universidad de Chile, Casilla 653, Santiago, Chile
}

Marcel G. Clerc ${ }^{\dagger}$

Departamento de Física, Facultad de Ciencias Físicas y Matemáticas, Universidad de Chile, Casilla 487-3, Santiago, Chile

(Received 12 April 2015; revised manuscript received 6 August 2015; published 15 October 2015)

\begin{abstract}
Stationary two-dimensional localized structures have been observed in a wide variety of dissipative systems. The existence, stability properties, dynamical evolution, and bifurcation diagram of an azimuthal symmetry breaking, rodlike localized structure in the isotropic prototype model of pattern formation, the Swift-Hohenberg model, is studied. These rodlike structures persist under the presence of nongradient perturbations. Interaction properties of the rodlike structures are studied. This allows us to envisage the possibility of different crystal-like configurations.
\end{abstract}

DOI: 10.1103/PhysRevE.92.042915

PACS number(s): 05.45.Yv, 89.75.Kd

\section{INTRODUCTION}

Macroscopic systems under the influence of injection and dissipation of energy, momenta, and matter often lead to the formation of spatial structures [1-3]. These patterns can be extended, involving the whole physical system, or localized, which are patterns that exist only on a portion of the system [4-6]. From the dynamical systems point of view, one-dimensional localized structures are homoclinic connections of the stationary dynamical system involving a stable and an unstable manifold of a given equilibrium $[7,8]$. The possibility of coexistence between different equilibria enriches the variety of possible homoclinic structures. For example, in the case of coexistence between a uniform and a pattern state, the heteroclinic entanglement generates the nucleation of a family of localized structures [8,9], which are organized by a snaking bifurcation diagram [10,11]. In recent decades, localized structures have been observed in different fields, such as magnetic materials [12], chemical reactions [13], vertically driven Newtonian fluid [14,15], granular media [16,17], liquid crystals [18], liquid crystal light valve [19-21], colloidal fluids [22], electrical discharges [23], thermal convection [24,25], and nonlinear optics [26,27], to mention a few. In most of these observations the localized states are two-dimensional objects with circular symmetry. Localized structures are particle-type solutions for nonlinear equations, as they exhibit a series of characteristics often attributed to particles such as a size, a position, and a velocity defined by the parameters of the system, and an interaction law between them. Localized structures have attracted the interest of the scientific community because of their potential applications in optical information storage and processing [28].

Homogeneous and isotropic systems-with translational and rotational invariance-usually exhibit localized patterns which are azimuthally symmetric, that is, the localized states have circular symmetry. Spatial breaking of symmetry tends to deform the localized structures and can even induce

\footnotetext{
*ibordeu@imperial.ac.uk

${ }^{\dagger}$ marcel@dfi.uchile.cl
}

propagation of them; this is the case of the worm structures observed in binary liquids [24,29] and electroconvection

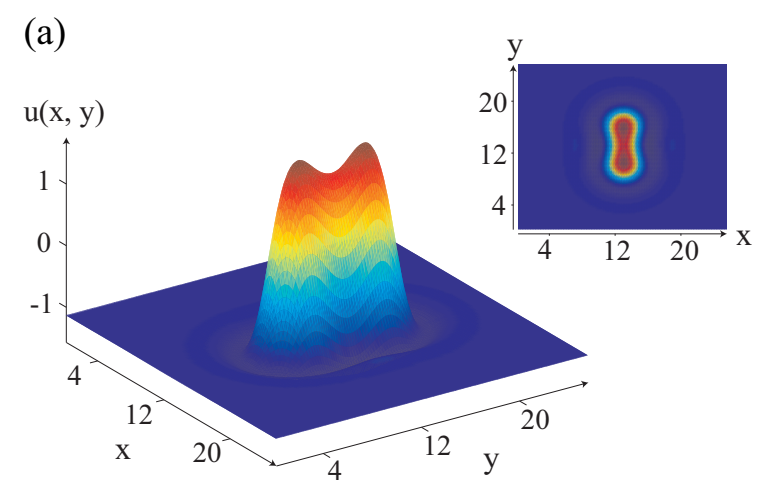

(b)

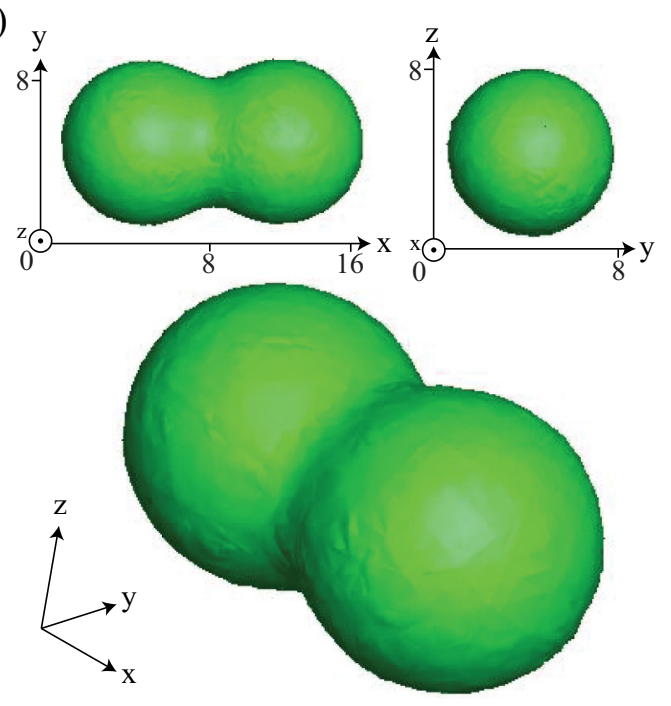

FIG. 1. (Color online) Stationary rod localized structure for (a) 2D Swift-Hohenberg model, Eq. (1), with $v=2.0, \eta=-0.355$, and $\epsilon=1.2$. The inset is a color map of the rod localized structure. (b) 3D Swift-Hohenberg model; image shows the isosurface for $u=0.2$ with $v=2.0, \eta=-0.37$, and $\epsilon=1.5$. Simulations in two and three dimensions were made using pseudospectral and adaptive triangular finite element methods, respectively. Both with Neumann boundary conditions for the field $u(\vec{r}, t)$. 
cells [30]. Theoretically, localized stripes [31], rolls, square, and worms have been observed in the two-dimensional Swift-Hohenberg equation [32]. In the liquid crystal light valve experiment triangular localized structures have been observed by controlling the optical feedback [20]. Numerical simulations of the model describing this system also exhibit this type of intriguing triangular localized state. Moreover, triangular localized structures are inherently two-dimensional due to the rotational symmetry breaking. There is no global geometric theory to explain the origin of these structures, and no characterization of the different possible localized structures without rotational symmetry has been done.

In this paper, we show the existence, stability properties, dynamical evolution, and bifurcation diagram of an elongated localized structure, nonazimuthally symmetric, in a prototype isotropic two-dimensional (2D) model, the Swift-Hohenberg equation. We call this object a rod localized structure. Figure 1 illustrates the typical observed rod structure in 2D and 3D systems. For 2D structures, we show the complex interaction scenario of rod structures, which have a complex network of equilibria. This allows the existence of different crystal-like configurations.

The paper is organized as follows: In Sec. II, the generalized Swift-Hohenberg model and its dynamical evolution features are introduced. In particular, the phase space of the model used is described. In Sec. III, the rodlike localized structure is analyzed as a composition of one-dimensional structures. The different instabilities and bifurcation diagrams of rod structures are analyzed in Sec. IV. Interaction properties of rod structures are shown in Sec. V. The persistence of the structure under nonvariational perturbations is studied in Sec. VI. Our conclusions and remarks are left to the final section.

\section{GENERALIZED SWIFT-HOHENBERG EQUATION}

Let us consider a prototype model which exhibits both localized and extended patterns. This is a natural variant of the Swift-Hohenberg equation [33], which is an isotropic, reflection symmetry, and real order parameter nonlinear equation deduced originally to describe the pattern formation on Rayleigh-Bénard convection [33]. This generalization includes an extra term which breaks the field reflection symmetry. It has been deduced in various field on nonlinear science such as chemistry [34], plant ecology [35], and nonlinear optics $[36,37]$. This equation applies to a wide range of systems that undergo a symmetry breaking instability-often called Turing instability $[2,4]$ — close to a second-order critical point marking the onset of a hysteresis loop, which corresponds to a Lifshitz point $[4,38,39]$. The generalized Swift-Hohenberg equation reads

$$
\frac{\partial u}{\partial t}=\eta+\epsilon u-u^{3}-v \nabla^{2} u-\nabla^{4} u
$$

where $u=u(x, y, t)$ is a real scalar field, $x$ and $y$ are spatial coordinates, and $t$ is time. Depending on the context in which this equation has been derived, the physical meaning of the field variable $u(x, y, t)$ could be the electric field, deviation of molecular orientations, phytomass density, or chemical concentration. The control or the bifurcation parameter $\epsilon$ measures the input field amplitude, the aridity parameter, or chemical concentration. The $\eta$ parameter breaks the reflection symmetry $u \rightarrow-u$, thus it accounts for the asymmetry between homogeneous states. When this parameter vanishes $(\eta=0)$, one recovers the original Swift-Hohenberg equation [33]. The parameter $v$ stands for the diffusion coefficient; when this parameter is negative $(v>0)$, it induces an antidiffusion process. The 2D Laplacian operator $\nabla^{2}=\partial_{x x}^{2}+\partial_{y y}^{2}$ and the 2D bilaplacian operator $\nabla^{4}$ act on the plane $(x, y)$. Thus the first three terms on the right hand side of Eq. (1) account for homogeneous or local nonlinear dynamics, the fourth and the fifth terms stand for the transport mechanisms or spatial coupling via diffusion and hyperdiffusion, respectively. In the 3D case [see Fig. 1(b)] the Laplacian and bilaplacian operators act on the whole $(x, y, z)$ space.

It should be noted that by a displacement of the field $u \rightarrow u+u_{0}$, where $u_{0}$ is a constant, Eq. (1) can be rewritten by removing the parameter $\eta$ and including an extra term proportional to $u^{2}$. The equation including the quadratic term has been widely studied in various contexts (see the textbook [3], and references therein). An important property of Eq. (1) is that it possess a gradient form, i.e.,

$$
\frac{\partial u}{\partial t}=-\frac{\delta F\left[u, \nabla u, \nabla^{2} u\right]}{\delta u},
$$

with the functional

$F \equiv \iint_{\mathbb{R}^{2}}\left(-\eta u-\epsilon \frac{u^{2}}{2}+\frac{u^{4}}{4}-v \frac{(\nabla u)^{2}}{2}+\frac{\left(\nabla^{2} u\right)^{2}}{2}\right) d x d y$.

Note that using the solutions of Eq. (1), this functional satisfies

$$
\frac{d F}{d t}=-\iint_{\mathbb{R}^{2}} d x d y\left(\partial_{t} u\right)^{2} \leqslant 0 .
$$

Hence, $F$ is a Lyapunov functional that can only decrease in the course of time. This property guarantees that with temporal evolution functional proceeds toward the state for which it has the smallest possible value which is compatible with the system's boundary conditions. Any initial distribution $u(x, y, t)$ [or $u(x, y, z, t)$ in the 3D case] evolves towards a homogeneous or inhomogeneous (periodic or localized) stationary state corresponding to a local or global minimum of $F$. The analysis of the functional $F$ is provided in Ref. [40].

The generalized Swift-Hohenberg equation exhibits coexistence between homogeneous and pattern states [41,42], thus allowing the stability of localized structures. These are localized structures in the sense of integral boundedness,

$$
\iint_{\mathbb{R}^{2}}\left|u_{l s}(x, y)\right|^{2} d x d y<+\infty
$$

where $u_{l s} \equiv u(x, y)-u_{0}$ is the relative field of the localized structure with respect to the homogeneous state $u_{0}$, which sustains the localized structure. This homogeneous state is a stable solution to the cubic equation $\eta+\epsilon u_{0}-u_{0}^{3}=0$. For a certain range of parameters $\{\eta, \epsilon\}$ two different localized structures are stable; the first is the well known circular (azimuthally 


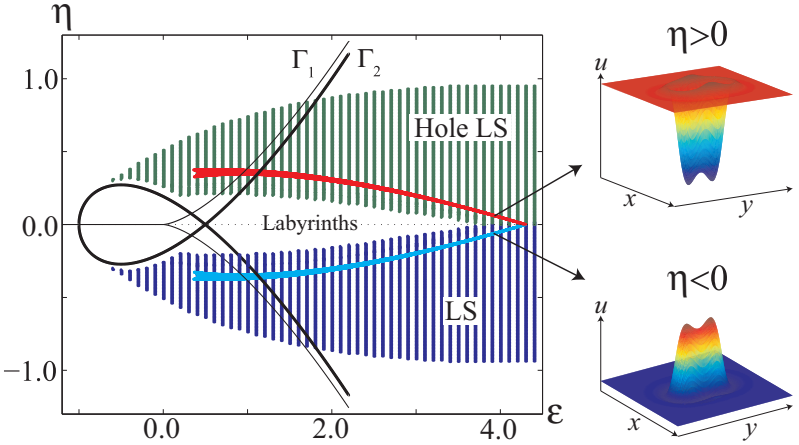

FIG. 2. (Color online) Bifurcation diagram of the $2 \mathrm{D}$ generalized Swift-Hohenberg Eq. (1) in $(\epsilon, \eta)$ space for $v=2.0$. The light and dark curve $\Gamma_{1}$ and $\Gamma_{2}$ account for the nascent bistability cycle and the spatial bifurcation of the uniform state, respectively. The shaded areas account for the zones where localized peaks (LS zone) and localized holes (hole LS zone) are observed. The painted areas stand for the region where rod structures have been observed. The insets correspond to the typical monitored rod structures.

symmetric) localized structure [43]. Notwithstanding, we have found a second type of localized structure that corresponds to a different class of localized structures at least in two- and three-dimensional isotropic systems. This object is a stable rodlike localized structure; it breaks the azimuthal symmetry, remaining invariant only with respect to a rotation of $\pi$ around any axis on the $(x, y)$ plane which contains the center of the localized structure. Figure 1 shows the typical rodlike stable localized structures exhibited by both the 2D and 3D Swift-Hohenberg Eq. (1).

In order to figure out the conditions under which the rod structure emerges, the analysis of the bifurcation diagram must be constructed for the model under study. For a fixed value of the diffusion coefficient $v=2.0$, a typical bifurcation diagram of the model Eq. (1) in the parameter space $(\epsilon, \eta)$ is described by curves $\Gamma_{1}$ and $\Gamma_{2}$ in Fig. 2 . The curves $\Gamma_{1}$ and $\Gamma_{2}$ represent the pitchfork bifurcation and the threshold associated with a modulation (or pattern-forming or Turing) instability of the homogeneous state, respectively $[41,42]$. For negative $\epsilon$, the system has only one homogeneous steady state, the monostable region. For positive $\epsilon$ the system undergoes a bistable behavior between homogeneous steady states as result of the saddle-node bifurcation (cf. Fig. 2). Moreover as a result of the spatial instability of the uniform state (cf. Fig. 2), the system also exhibits coexistence between patterns and homogeneous states. Near this type of bistability region one expects to observe stable localized structures. The shaded zones in Fig. 2 account for the areas where stable 2D circular localized peaks and holes are observed. When one decreases the value of $|\eta|$ (approaching zero), localized structures become unstable giving rise to labyrinthine patterns [44]. This transition occurs via fingering instability. Unexpectedly, rod structures coexist with isotropic localized structures. By direct simulation of Eq. (1) we have uncovered the region where the rod structures are observed, see Fig. 2.

Even though the Swift-Hohenberg model has been extensively studied since its deduction, no analytic expression is know for the localized solution. This is because these structures are homoclinic solutions of the stationary dynamical system $\left(\partial_{t} u=0\right)$, which is chaotic when replacing time for the radial coordinate [45]. Under this consideration, the study of the rodlike structure will not lead to an analytic expression nor to a full characterization of its characteristic properties, bifurcations, and interaction. Thus, numerical and geometrical methods are the most suitable tools for characterizing the localized structures.

Simulations in the 2D case were implemented using a pseudospectral code, with a $512 \times 512$ points grid. Figure 1 (a) shows a subregion of $240 \times 240$ points, with spacing $d x=$ 0.1 . The structures do not align with the simulation axes, and can be positioned in any direction. In $2 \mathrm{D}$ simulations using the finite differences code with Runge-Kutta order-4 algorithm, rod structures are also observed, however the discretization of the square grid can induce the alignment of the rod structure with the simulation axes. For the $3 \mathrm{D}$ case, a triangular finite element code with adaptive spatial and temporal steps was used, and a simulation box of dimensions $40 \times 40 \times 40$ was considered. Figure 1(b) shows a zoomed region of size $16 \times 8 \times 8$. Here, rod localized structures also can be oriented in any direction.

\section{ONE-DIMENSIONAL INTERPRETATION}

No definitive theory for two-dimensional localized structures has yet been formulated, therefore, the required physical and mathematical conditions for their existence and stability are not known. However, for one-dimensional systems, localized structures emerge as a family of stable fronts connecting a homogeneous with a pattern state in a bistable regime [46]. It is now known that coexistence (instead of bistability) is sufficient for the appearance of one-dimensional localized structures [47]. In this sense, the generation of two-dimensional localized structures can be regarded as an extension of one-dimensional localized structure, which is rotated over its axis thus generating an azimuthally symmetric localized structure. Nevertheless, the rodlike structure has no azimuthal symmetry. We can project the 2D rod structure over two orthogonal planes $\left(\gamma_{1}\right.$ and $\left.\gamma_{2}\right)$. These projections generate the equivalent to one-dimensional localized structures. The projection over the $\gamma_{1}$ plane [Fig. 3(a)] generates a one wavelength wide localized structure while the projection over the $\gamma_{1}$ plane [Fig. 3(b)] generates a two wavelength wide localized structure [8]. Hence, the two-dimensional rod structure can be considered as the composition of two one-dimensional localized structures with different lengths.

As in one-dimensional localized structures of the generalized Swift-Hohenberg equation, the two-dimensional structures possess spatially oscillating tails of the field, which propagate radially from the bulk of the structure; these oscillations, that decay exponentially, stabilize the structure and allow the interaction between two or more of them by field interference $[48,49]$.

\section{INSTABILITIES AND BIFURCATIONS}

The characterization of the phase space of the generalized Swift-Hohenberg Eq. (1), Fig. 2, shows the existence of the rod structure for a narrow region of parameters which extends from 

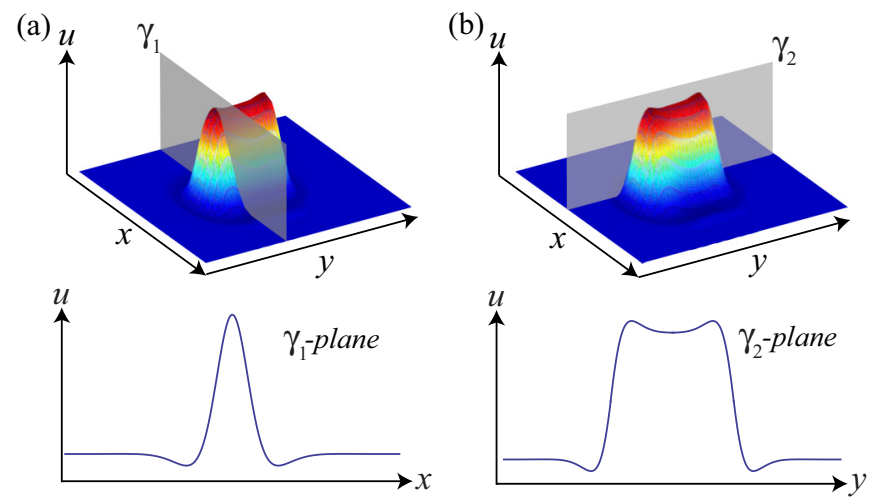

FIG. 3. (Color online) One-dimensional projections of the 2D rod structure over the (a) $(x, u)$ plane and (b) $(y, u)$ plane. Obtained by numerical simulation of Eq. (1) with $v=2.0, \eta=-0.355$, and $\epsilon=1.2$.

zones before the bistability $\Gamma_{1}$ curve up to values of $\epsilon \approx 4.3$. In the whole region of stability the rod structure coexists with stable localized spots. For $\eta=0$ only circular localized structures are observed. It has been shown that circular localized structures suffer from a curvature instability when leaving their stability zone thus generating an extended labyrinthine structure [44]. In this section is shown how labyrinths can emerge-in zones where circular localized structures are stable - by the destabilization of the rod structure. It is also shown how the rod structure elongates into an infinite roll structure, decays into the simpler localized spot, or even splits into a bound state of two circular localized structures, depending on the varied parameters. Bifurcations suffered by the rod structure can be studied through monitoring the energy (Lyapunov functional) while modifying one parameter and fixing the others.

By variations of the parameters $\eta$ or $\epsilon$ the rod structure is affected by saddle-node bifurcations characterized by a change on the energy which follows a square-root law near the threshold (see dotted lines in Figs. 5 and 7), and by a decay rate of the structure proportional to $\left(\alpha-\alpha_{c}\right)^{-1 / 2}$, where $\alpha$ is the varied parameter and $\alpha_{c}$ indicates the critical parameter value for which the bifurcation occurs [50]. Figure 4 illustrates this type of dynamical behavior. Rod structures that exist at the right side of the $\Gamma_{2}$ curve (cf. Fig. 2) exhibit only two different bifurcations. The first occur when leaving the stability zone of the rod structure by decreasing $\epsilon$ (or decreasing $|\eta|$ ), causing an increment in the rod structure's size and consequently an increment on its energy. Once the bifurcation takes place through the saddle-node mechanism, the system falls into the basin of attraction of a labyrinthine pattern. Figure 5 depicts the transition from rod to labyrinth by changing the different control parameters. As labyrinths are extended patterns, their energy diverges. The second bifurcation suffered by the rod structure in this zone takes place when increasing the value of $\epsilon$ (or increasing $|\eta|$ ); here, the rod structure shrinks, reducing its energy. By a saddle-node bifurcation, the rod structures decay into single localized spots, which are energetically more stable in the Lyapunov sense. Figure 5 shows the transition from rod to localized spots. This rod to circular structure bifurcation continues existing for values of $\eta$ and $\epsilon$ to the left of $\Gamma_{2}$

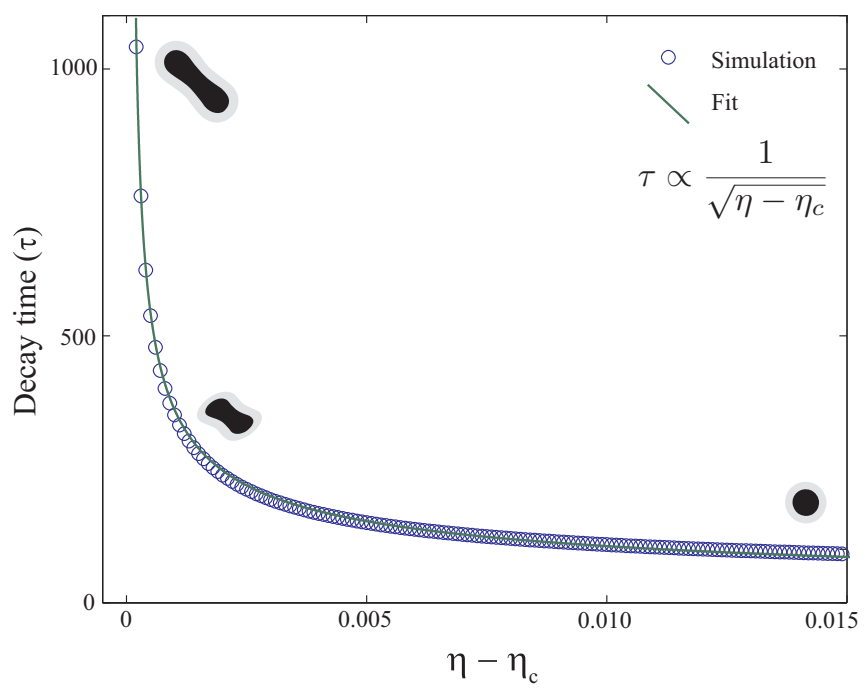

FIG. 4. (Color online) Decay rate as function of $\eta$ from a rod structure into a circular localized structure for the 2D generalized Swift-Hohenberg Eq. (1) with $\epsilon=1.6$ and $v=2.0$. The circles account for decay time obtained numerically. The solid curve is obtained using the expression $\tau=\tau_{0} / \sqrt{\eta-\eta_{c}}$ with fitting parameters $\eta_{c}=-0.3444$ and $\tau_{0}=11.61$.

curve. The decay rate from rod to localized spot is shown in Fig. 4, where $\epsilon=1.6$. The numeric decay rate law corresponds to the expected theoretical rate from saddle-node bifurcation theory [50].

Another scenario emerges for values of $\eta$ and $\epsilon$ to the left side of the $\Gamma_{2}$ curve. Another two bifurcations are observed when overstepping the boundaries of the stability zone of the rod structure. For fixed values of $\epsilon$, decreasing $|\eta|$ (see the transition $\zeta_{4}$ curve in Fig. 6), the rod structure exhibits a continuous elongation similar to the case of the rod to labyrinth bifurcation, though in this case the elongation is permanent generating an infinitely long stripe structure without transversal oscillations [see the inset in Fig. 5(b)]. A fourth bifurcation appears when leaving the stability zone of the rod structure by the interior of the horseshoelike arc. Figure 6 shows a zoomed phase diagram for the rod structure. By following the $\zeta_{5}$ curve, the rod structure becomes unstable; surface tension is unable to keep the structure together leading to its splitting. Figure 7 depicts the bifurcation observed in the horseshoelike zone. Through this bifurcation two circular localized structures are generated by the collapse of the central part of the rod structure.

It is important to note that there is no transition from circular to rod structure, as the circular structure always has a lower energy. However, transitions from labyrinthine to rod structures are observed when entering with a labyrinth to the stability zone of the rod structure.

\section{INTERACTION PROPERTIES}

It has been shown that the generalized Swift-Hohenberg Eq. (1), allows the existence of multiple stable localized structures [43]. These (one-, two-, or three-dimensional) structures possess no compact support, thus the frontier between the homogeneous state $u_{0}$ and the localized structure 
(a)

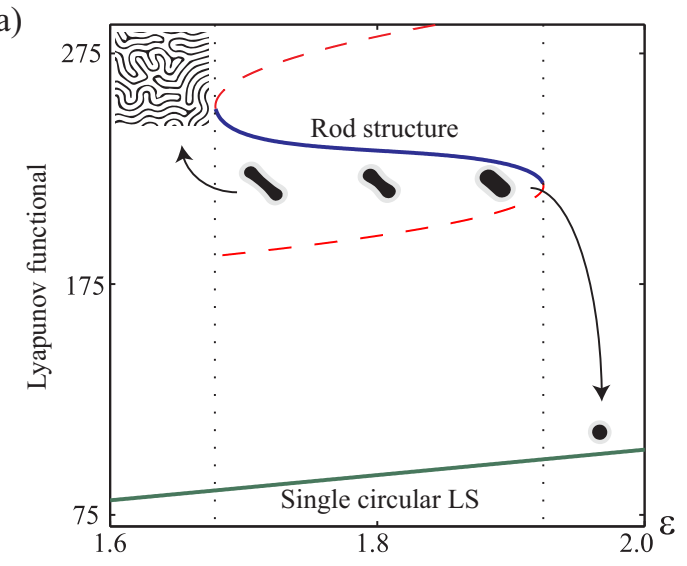

(b)

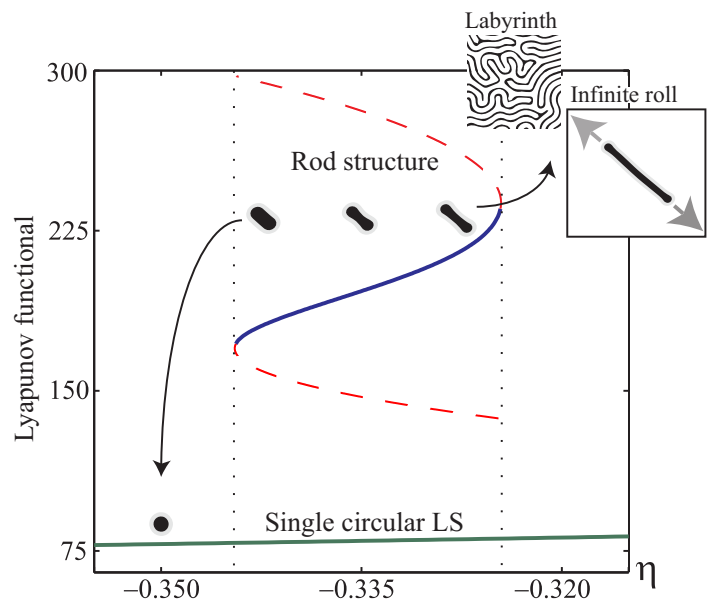

FIG. 5. (Color online) Bifurcation diagrams of rod structures as function of $\epsilon$ and $\eta$ parameters obtained by direct simulation of Eq. (1). Bifurcations observed: rod to circular localized structure, rod to labyrinth, and rod to infinite roll. For (a) $\eta=-0.320$ and (b) $\epsilon=1.6$, with $v=2.0$. The dotted red lines represent the square-root change rate of the energy, characteristic of saddle-node bifurcations.

is not defined. Instead, the field oscillates decaying exponentially with the distance from the localized structure, these oscillations fluctuate around the homogeneous state with the characteristic wavelength of the system. The exponential tail will be addressed as the interaction field $[15,49,51,52]$.

As mentioned before, no analytical expression is know for the localized structures in the generalized Swift-Hohenberg model. Nevertheless, for interaction properties, only the asymptotic approximation of the decaying field is relevant. Based on the linear perturbation theory, it has been shown [49] that a circular localized structure possesses an interaction field that projects radially from the structure, with an oscillatory behavior describing the existence attracting and repelling forces.

A system with two (or more) localized structures positioned randomly within the system, will evolve towards a stationary equilibrium by changes in the relative positions between the structures. The interaction fields corresponding to each particle interfere, exerting forces, which in turn induce movement of the particlelike solutions.
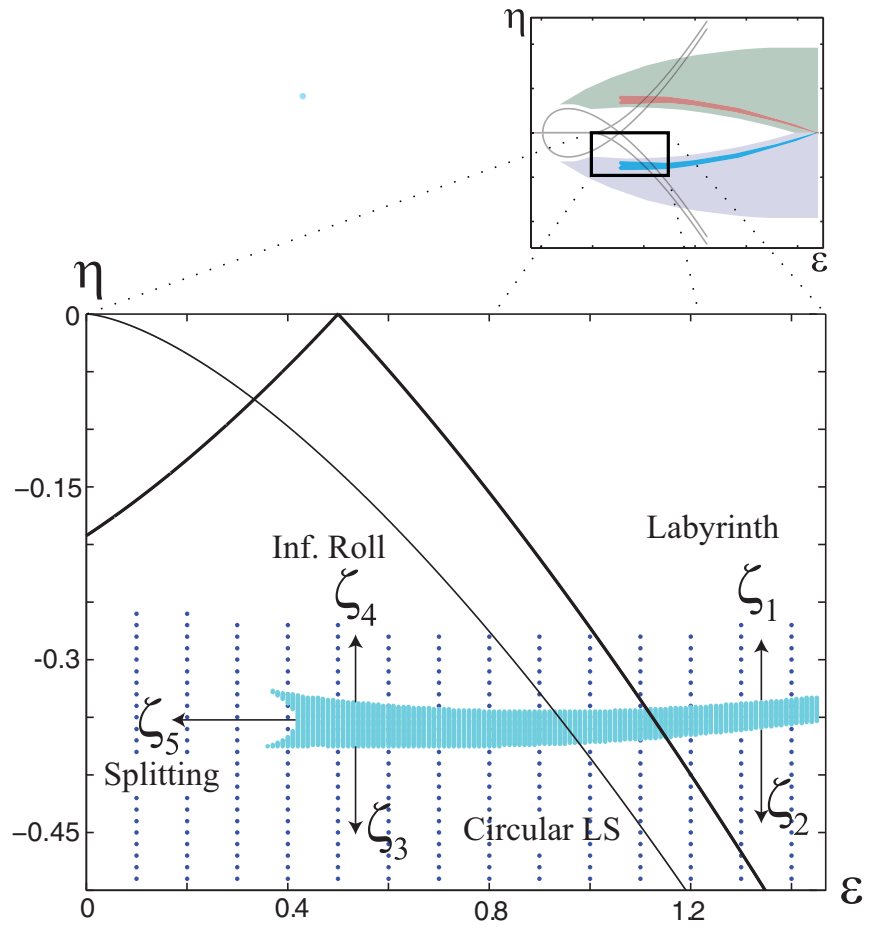

FIG. 6. (Color online) Zoomed region of the phase diagram, which shows the different outcomes possible from the destabilization of a rod structure. $\zeta_{1}$ curve indicates the transition from rod to labyrinth bifurcation, $\zeta_{2}$ and $\zeta_{3}$ curves account for the transition between rod and circular localized structure, $\zeta_{4}$ curve stands for the transition from rod to infinite roll, and $\zeta_{5}$ curve accounts for the transition between rod and binary state, for $v=2.0$.

\section{Rod LS interaction characterization}

The analytical study of the interaction field of rod structures requires the derivation of the asymptotic field for the rod structure, which must include an azimuthal dependency given by the shape of the structure; not having this information makes the calculations nonviable.

Through direct simulations of Eq. (1), we were able to evaluate the several stationary equilibrium points of the

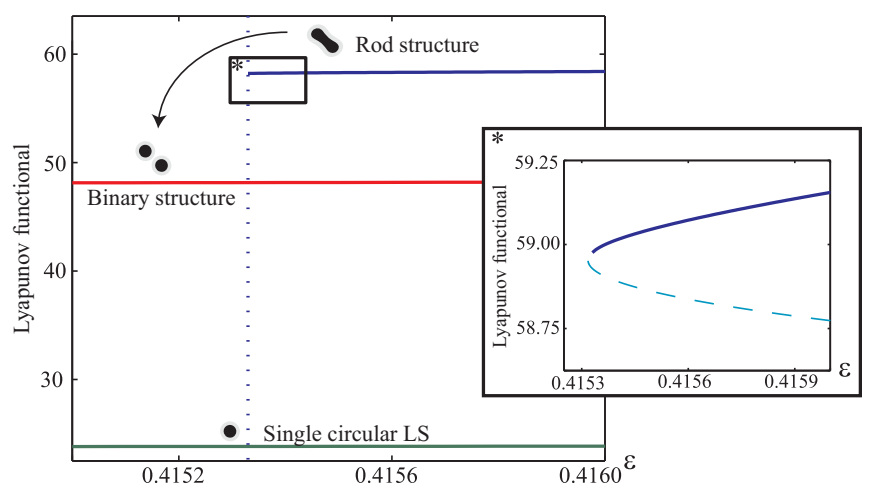

FIG. 7. (Color online) Bifurcations for the splitting of a rod structure into a binary structure, obtained by direct simulation of the generalized Swift-Hohenberg Eq. (1) for $v=2.0, \eta=-0.350$. The inset corresponds to a zoom of the bifurcation point, the dotted line represents the square-root fit, characteristic of the saddle-node bifurcation. 

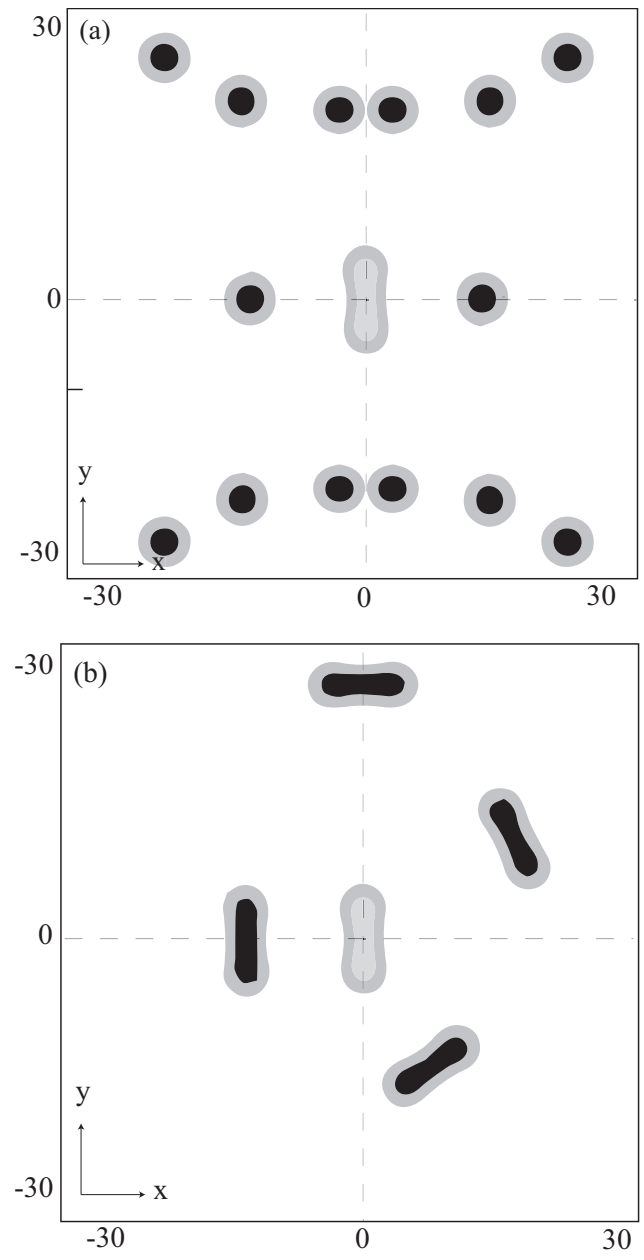

FIG. 8. Several stationary equilibrium positions for (a) rod-LS interaction, and (b) rod-rod interaction. Numerically obtained by simulations of the Swift-Hohenberg model, Eq. (1), with $v=2.0$, $\eta=-0.355, \epsilon=1.2$, and specular boundary condition, considering only two structures at a time; central rod structure is considered static.

rod-circular and rod-rod structure interaction. Considering a central rod structure (see Fig. 8, gray structure), and a circular LS with a random position away from the rod, the systems evolves towards a stationary equilibrium given by the stationary relative position between particles. These equilibriums are shown in Fig. 8(a).

More complex is the rod-rod interaction, for their azimuthal asymmetry reflected on its axial elongation. This adds an angular degree of freedom for the positioning of a rod structure at each equilibrium point [see Fig. 8(b)]. The variety of equilibriums exhibited by this type of structures allows the existence of diverse complex arrangements when multiple rod structures are considered. The interaction of a large number of rods (i.e., covering all the available space) increases the number of possible equilibrium configurations. The multiple interactions may drive the system towards equilibriums which were unstable in the rod-rod interaction scenario; in Figs. 9(a)9(c), dashed lines indicate rod-rod equilibriums which are unstable in an isolated environment and stabilize under the presence of multiple structures; the use of periodic boundary conditions is important for the stabilization of these lattices.
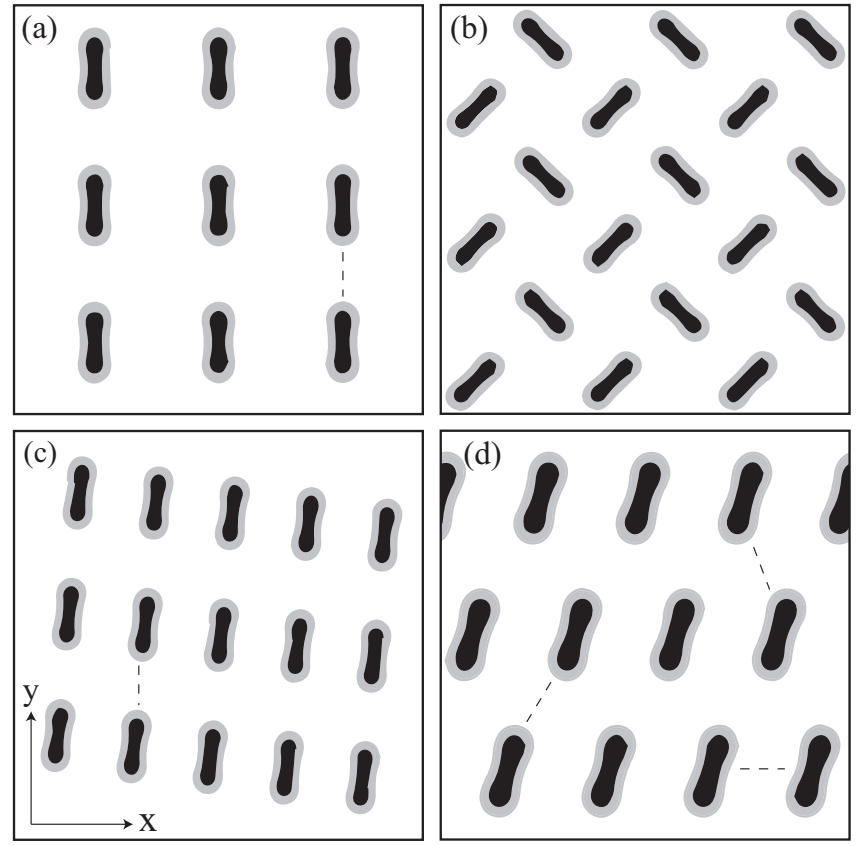

FIG. 9. Tailored crystal-like configurations generated by the collocation of rod structures for the Swift-Hohenberg model, Eq. (1), with $v=2.0, \eta=-0.355, \epsilon=1.2$ and periodic boundary conditions. Simulation grid of $256 \times 256$ points, and spacing $d x=0.25$ for (a), (b), and (c), and $d x=0.2$ for (d).

The crystal-like structure shown in Fig. 9(d) is constructed based on the T-like equilibrium position, orthogonal rod structures, exhibited by the rod-rod interaction. These lattices persist under noise perturbations.

\section{NONVARIATIONAL PERTURBATIONS}

The generalized Swift-Hohenberg model, Eq. (1), has a nonvariational extension deduced in the context of liquid crystals for bouncing localized states [19] and deduced from chemical, biological, and optical models [53]. This equation (Lifshitz normal form) reads

$$
\frac{\partial u}{\partial t}=\eta+\epsilon u-u^{3}-v \nabla^{2} u-\nabla^{4} u+b u\left(\nabla^{2} u\right)+c(\nabla u)^{2},
$$

which (excluding the case where $b=2 c$ ) is nonvariational, thus it cannot be derived from a Lyapunov functional. This allows the model to exhibit complex and permanent behaviors such as oscillations, chaos, and other spatiotemporal dynamics. In one spatial dimension a detailed study of localized states of this nonvariational Swift-Hohenberg equation is presented in Ref. [54]. The last two terms of Eq. (6) correspond, respectively, to nonlinear diffusion, $b$ being the nonlinear diffusion coefficient, and nonlinear advection.

Numerically, we have observed that for small ranges of values of the parameters $b$ and $c$, the rod structure remains stable only by changes on its dimensions. For constant values of $\epsilon$ and $\eta$, increasing $b$ or decreasing $c$ affects the structure, by making it shorter, while decreasing $b$ or increasing $c$ enlarges the structure. For parameters $b$ and $c$ where the rod structures 
are larger, the evolution of the system is faster; for instance, the formation of labyrinthine patterns takes less time.

For fixed values of the parameters, $\epsilon=1.2, \eta=-0.355$, and $v=2.0$ the range of $b$ and $c$ for which the rod structure is stable is approximately $b=[-0.017,0.015]$ and $c=[-0.007,0.008]$. Despite the small range of $b$ and $c$ for which the rod is stable, the possibility of existence of rod structures in nonvariational systems opens the possibility for searching this structure in a wider variety of experiments.

\section{CONCLUSIONS}

An asymmetric localized solution for the isotropic generalized Swift-Hohenberg model in two and three spacial dimensions has been found. This solution called rod or rodlike localized structure breaks the azimuthal symmetry, remaining invariant only with respect to a rotation of $\pi$ around any axis on the $(x, y)$ plane which contains the center of the localized structure. The existence, bifurcation diagram, stability properties, and interaction have been addressed. The questions of whether this type of solution can be observed experimentally or if it exists in other isotropic systems remain open.

For a simple nonvariational generalization of the SwiftHohenberg equation, the Lifshitz normal form, we have shown that the rodlike localized structures persist. Likewise, generalizations of the Swift-Hohenberg model, which may include other types of nonlinearity or nonvariational or other isotropic terms, it is expected that the rodlike structure must persist in a given range of parameters. Indeed, this is a consequence of the robust nature of the localized states.

\section{ACKNOWLEDGMENTS}

M.G.C. acknowledges the financial support of FONDECYT Project No. 1150507. I.B. was supported by CONICYT, scholarship Beca de Magister Nacional No. 22130947.
[1] P. Glansdorff and I. Prigogine, Thermodynamic Theory of Structures, Stability and Fluctuations (Wiley, New York, 1971).

[2] G. Nicolis and I. Prigogine, Self-Organization in Non Equilibrium Systems (Wiley, New York, 1977).

[3] L. M. Pismen, Patterns and Interfaces in Dissipative Dynamics (Springer, Berlin, 2006).

[4] M. C. Cross and P. C. Hohenberg, Rev. Mod. Phys. 65, 851 (1993).

[5] H. G. Purwins, H. U. Bodeker, and S. Amiranashvili, Adv. Phys. 59, 485 (2010).

[6] O. Descalzi, M. Clerc, S. Residori, and G. Assanto, Localized States in Physics: Solitons and Patterns (Springer, New York, 2011).

[7] W. van Saarloos and P. C. Hohenberg, Phys. Rev. Lett. 64, 749 (1990).

[8] P. Coullet, Int. J. Bifurcation Chaos 12, 2445 (2002).

[9] P. Coullet, C. Riera, and C. Tresser, Phys. Rev. Lett. 84, 3069 (2000).

[10] P. D. Woods and A. R. Champneys, Physica D 129, 147 (1999).

[11] F. Haudin, R. G. Rojas, U. Bortolozzo, S. Residori, and M. G. Clerc, Phys. Rev. Lett. 107, 264101 (2011).

[12] H. A. Eschenfelder, Magnetic Bubble Technology (SpringerVerlag, Berlin, 1981).

[13] K.-Jin Lee, W. D. McCormick, J. E. Pearson, and H. L. Swinney, Nature (London) 369, 215 (1994).

[14] J. Wu, R. Keolian, and I. Rudnick, Phys. Rev. Lett. 52, 1421 (1984).

[15] M. G. Clerc, S. Coulibaly, N. Mujica, R. Navarro, and T. Sauma, Philos. Trans. R. Soc. A 367, 3213 (2009).

[16] P. Umbanhowar, F. Melo, and H. Sweeney, Nature (London) 382, 793 (1996).

[17] M. G. Clerc, P. Cordero, J. Dunstan, K. Huff, N. Mujica, D. Risso, and G. Varas, Nat. Phys. 4, 249 (2008).

[18] S. Pirkl, P. Ribiere, and P. Oswald, Liq. Cryst. 13, 413 (1993).

[19] M. G. Clerc, A. Petrossian, and S. Residori, Phys. Rev. E 71, 015205 (2005).
[20] U. Bortolozzo, L. Pastur, P. L. Ramazza, M. Tlidi, and G. Kozyreff, Phys. Rev. Lett. 93, 253901 (2004).

[21] U. Bortolozzo, M. G. Clerc, C. Falcon, S. Residori, and R. Rojas, Phys. Rev. Lett. 96, 214501 (2006).

[22] O. Lioubashevski, Y. Hamiel, A. Agnon, Z. Reches, and J. Fineberg, Phys. Rev. Lett. 83, 3190 (1999).

[23] Y. A. Astrov and Y. A. Logvin, Phys. Rev. Lett. 79, 2983 (1997).

[24] R. Heinrichs, G. Ahlers, and D. S. Cannell, Phys. Rev. A 35, R2761 (1987).

[25] P. Kolodner, D. Bensimon, and C. M. Surko, Phys. Rev. Lett. 60, 1723 (1988).

[26] F. T. Arecchi, S. Boccaletti, and P. L. Ramazza, Phys. Rep. 318, 1 (1999).

[27] B. Schapers, M. Feldmann, T. Ackemann, and W. Lange, Phys. Rev. Lett. 85, 748 (2000).

[28] S. Barland, J. R. Tredicce, M. Brambilla, L. A. Lugiato, S. Balle, M. Giudici, T. Maggipinto, L. Spinelli, G. Tissoni, T. Knödl, M. Miller, and R. Jäger, Nature (London) 419, 699 (2002).

[29] E. Moses, J. Fineberg, and V. Steinberg, Phys. Rev. A 35, 2757 (1987).

[30] M. Dennin, G. Ahlers, and D. S. Cannell, Phys. Rev. Lett. 77, 2475 (1996).

[31] M. G. Clerc, D. Escaff, and R. Rojas, Europhys. Lett. 83, 28002 (2008).

[32] D. Lloyd, J. Burke, E. Knobloch, and B. Sandstede, SIAM J. Math. Anal. 41, 936 (2009).

[33] J. Swift and P. C. Hohenberg, Phys. Rev. A 15, 319 (1977).

[34] M'F. Hilali, G. Dewel, and P. Borckmans, Phys. Lett. A 217, 263 (1996).

[35] R. Lefever, N. Barbier, P. Couteron, and O. Lejeune, J. Theor. Biol. 261, 194 (2009).

[36] M. Tlidi, M. Georgiou, and P. Mandel, Phys. Rev. A 48, 4605 (1993).

[37] J. Lega, J. V. Moloney, and A. C. Newell, Phys. Rev. Lett. 73, 2978 (1994). 
[38] L. D. Landau and E. M. Lifshitz, Statistical Physics, 2nd ed. (Pergamon, New York, 1968), Chap. XIV.

[39] R. M. Hornreich and M. Luban, Phys. Rev. Lett. 35, 1678 (1975); R. M. Hornreich, J. Magn. Magn. Mater. 15, 387 (1980).

[40] A. G. Vladimirov, R. Lefever, and M. Tlidi, Phys. Rev. A 84, 043848 (2011).

[41] P. Coullet, C. Riera, and C. Tresser, Prog. Theor. Phys. Supp. 139, 46 (2000).

[42] F. del Campo, F. Haudin, R. G. Rojas, U. Bortolozzo, M. G. Clerc, and S. Residori, Phys. Rev. E 86, 036201 (2012).

[43] M. Tlidi, P. Mandel, and R. Lefever, Phys. Rev. Lett. 73, 640 (1994)

[44] I. Bordeu, M. G. Clerc, R. Lefever, and M. Tlidi, Commun. Nonlinear Sci. Numer. Simul. 29, 482 (2015).

[45] P. Coullet, C. Elphick, and D. Repaux, Phys. Rev. Lett. 58, 431 (1987).
[46] M. G. Clerc and C. Falcon, Physica A 356, 48 (2005).

[47] U. Bortolozzo, M. G. Clerc, and S. Residori, New J. Phys. 11, 093037 (2009).

[48] J. Burke and E. Knobloch, Phys. Rev. E 73, 056211 (2006).

[49] I. Aranson, K. Gorshkov, A. Lomov, and M. Rabinovich, Physica D 43, 435 (1990).

[50] S. H. Strogatz, Nonlinear Dynamics and Chaos: With Applications to Physics, Biology, Chemistry and Engineering (Addison-Wesley, Reading, MA, 1994).

[51] K. Kawasaki and T. Otha, Phys. A (Amsterdam, Neth.) 116, 573 (1982).

[52] M. G. Clerc, S. Coulibaly, and D. Laroze, Europhys. Lett. 90, 38005 (2010).

[53] G. Kozyreff and M. Tlidi, Chaos 17, 037103 (2007).

[54] J. Burke and J. H. Dawes, SIAM J. Appl. Dyn. Syst. 11, 261 (2012). 